\title{
Wireless Mesh Router Placement with Constraints of Gateway Positions and QoS
}

\author{
Chun-Cheng Lin, Tung-Huei Chen, Shun-Yu Jhong \\ Department of Industrial Engineering and Management, National Chiao Tung University, Hsinchu 300, Taiwan \\ E-mails: cclin321@nctu.edu.tw,iamhuei01@gmail.com,jsyu0527@gmail.com
}

\begin{abstract}
The past studies on router node placement for wireless mesh networks (WMNs) did not consider placement of Internet gateways. Therefore, mesh routers and mesh clients can only communicate locally. The problem in this paper is to maximize both network connectivity and client coverage for the router node placement in WMNs consisting of mesh routers, mesh clients, and Internet gateways, subject to three QoS constraints: delay, relay load, and Internet gateway capacity. By visualizing the placements in previous works, we discover two main drawbacks: overlapping and coverless. To solve them, this paper presents a novel particle swarm optimization approach. Performance of the proposed approach is verified by simulation.
\end{abstract} QoS

Keywords - Wireless mesh network, particle swarm optimization,

\section{INTRODUCTION}

In general, router node placement (RNP) in wireless mesh networks (WMNs) are divided into two categories: mesh router nodes placement [1] and Internet gateway node placement [2]. The former RNP problem is considered in static WMNs [3] and dynamic WMNs [4]. The main difference is that in dynamic WMNs both mesh clients and mesh routers have mobility and mesh clients can switch on or off their network access at different times. In order to serve more mesh clients by placement of mesh routers, the problem aims to achieve the maximal network connectivity and client coverage. For this type of problems, generally heuristic algorithms are used, such as genetic algorithm, particle swarm optimization method, etc. The second category of RNP problems, Internet gateways nodes placement problem, is posed by Internet gateways and mesh routers, and aims to find the minimal number of Internet gateways and delay-hops for lower cost.

Since the previous studies on mesh router nodes placement only considered the WMNs with mesh routers and mesh clients, such local area networks cannot access to the Internet. On the other hand, since the previous studies on Internet gateway node placement only considered WMNs consisting of Internet gateways and mesh routers, the resultant placement without mesh clients cannot model the behavior of mesh clients. Hence, this paper proposes a new problem of mesh routers node placement in wireless mesh networks, which incorporates the above two problems. The concerned WMNs consist of three types of nodes: Internet gateway, mesh routers and mesh clients. In addition of including Internet gateways, the WMNs in this paper considers three QoS constraints: delay constraints, relay constraints, and capacity constraints.

\section{PROBLEM DESCRIPTION}

WMNs in this paper consists of three types of nodes: Internet gateways, mesh routers, and mesh clients. Each of Internet gateways and mesh routers has wireless coverage of a different size. Internet gateways can provide network services to mesh clients and mesh routers within the coverage range. Each mesh client can access the Internet if it is located within the coverage of a mesh router that has accessed to an Internet gateway directly or indirectly. Variable used in the concerned problem is given as follows:

- $\quad U$ : Set of all nodes.

- $I G W$ : Set of Internet gateways.

- $R$ : Set of mesh routers.

- $C$ : Sect of mesh clients.

- $\quad D(v)$ : Placement of node $v$.

- $\gamma_{v}$ : Size of radio coverage range of node $v$.

- $E$ : Set of links.

- $\Upsilon_{\text {node }}$ : Coverage range of the placement of the node.

- $G$ : The network topology.

The problem setting in this paper continues that in [4]. Consider a WMN with $w$ Internet gateways, $n$ mesh routers and $m$ mesh clients deployed in a two-dimensional geographical area of size $W \times H$. Let the mesh nodes in the WMN be denoted by $U=I G W \cup R \cup C$ in which

- $I G W=\left\{i g w_{1}, i g w_{2}, \ldots, i g w_{w}\right\}$ where each $i g w_{i}$ represents an Internet gateway and has radio coverage of size $\gamma_{i g w_{i}}$.

- $R=\left\{r_{1}, r_{2}, \ldots, r_{n}\right\}$ where each $r_{i}$ represents a mesh router and has a radio coverage range of size $\gamma_{r_{i}}$.

- $C=\left\{c_{1}, c_{2}, \ldots, c_{m}\right\}$ where each $c_{i}$ represents a mesh client.

This paper considers a WMN scenario in which Internet gateways are predetermined because they are connected with the Internet/wired backbone. According to the above, each mesh client is located at $D\left(c_{i}\right) \in \mathrm{R}^{2}$ in the deployment area. The positions of mesh routers are placed according to the deployment of each mesh client, denoted by $D(R)=\left\{D\left(r_{1}\right)\right.$, $\left.D\left(r_{2}\right), \ldots, D\left(r_{n}\right)\right\}$. And the placement of Internet gateways are fixed positions, denoted by $D(I G W)=\left\{D\left(i g w_{1}\right), D\left(i g w_{2}\right), \ldots\right.$, $\left.D\left(i g w_{w}\right)\right\}$. Let the circle centered at location $D\left(i g w_{i}\right)$ of node $i g w_{i}$ with radius size $\gamma_{i g w_{i}}$ be denoted by $\Upsilon_{i g w_{i}}$. And the node of mesh router $r_{i}$ is denoted by $\Upsilon_{r_{i}}^{t}$. For a determined placement of mesh routers, we can model a topology graph $G=(U, E)$ in which 
- $U=I G W \cup R \cup C$.

- for any Internet gateway $i g w_{i} \in I G W$ and any mesh router $r_{j} \in R$, edge $\left(i g w_{i}, r_{j}\right) \in E$, if $\Upsilon_{i g w_{j}} \cap \Upsilon_{r_{j}}=\varnothing$.

- for any two mesh routers $r_{i}, r_{j} \in R$, one of them has been accessing to Internet gateway, while the other one is not. edge $\left(r_{i}, r_{j}\right) \in E$, if $\Upsilon_{r_{i}} \cap r_{r_{j}}=\varnothing$.

- for any Internet gateway $i g w_{i} \in I G W$ and any mesh client $c_{j} \in C$, edge $\left(i g w_{i}, c_{j}\right) \in E$ if $D_{t}\left(c_{j}\right) \in \Upsilon_{i g w_{i}}$ and $d\left(c_{i}\right)=$ 0 , where $d_{t}\left(c_{i}\right)=0$ is meaning that $c_{i}$ does not link other node, mesh router or Internet gateway, if be linking is $d\left(c_{i}\right)$ $=1$.

- for any mesh client $c_{j} \in C$ and any mesh router $r_{j} \in R$, edge $\left(c_{i}, r_{j}\right) \in E$, if $D\left(c_{i}\right) \in \Upsilon_{r_{i}}$ and $d\left(c_{i}\right)=0$.

The WMN topology graph $G$ may not be connected, i.e., graph $G$ could consist of some subgraph components. However, the connectivity is mainly determined by the number of Internet gateways, because mesh routers must be linked to some Internet gateway to get access to the Internet. Assume that graph $G$ has $h$ subgraphs components $G_{1}, \ldots, G_{q}, \ldots, G_{h}$ in $G_{t}$, i.e., $G=G_{1} \cup G_{2} \cup \cdots \cup G_{h}$ and $G_{i} \cap G_{j}=\varnothing$, for $i, j \in\{1, \ldots, h\}, i \neq j$; and each of $G_{1}, \ldots, G_{q}$ is a subgraph component linked to some Internet gateway, but the other subgraph components are not. The first objective to measure performance of the WMN placement is the network connectivity, which is measured by size of the greatest subgraph component in $G$ as modelled as follows:

$$
\delta(G)=\sum_{i \in\{1,2 \ldots, q\}}\left\{\left|G_{i}\right|\right\}
$$

The second objective is the client coverage, which can be expressed as follows:

$$
\phi(G)=\left\{\left\{i ; d\left(c_{i}\right)=1 \text { for } i \in\{1, \ldots, m\}\right\} \mid\right.
$$

In addition to the above QoS concerns that are based on our previous work, this paper further considers positons of Internet gateways and QoS constraints [5]. Consider the following three QoS constraints, for delay hops, relay load, and Internet gateway capacities, respectively:

- Delay hop $D_{\text {hop }}$ is defined as the maximal acceptable number of hops from a mesh router to an Internet gateway.

- Relay load $R_{M R s}$ is defined as the upper bound of number of nodes that a node can be linked with, i.e., degree of the node in the topology graph.

- Internet gateway capacity $C_{M R s}$ (resp., $C_{M C S}$ ) is defined as the upper bound of numbers of mesh routers (resp., mesh clients) that an Internet gateway can serve.

This paper models the concerned problem as an integer programming model [6] with two objectives: network connectivity $\delta(G)$ and client coverage $\phi(G)$ subject to QoS constraints. Two decision variables are defined as follows:
$I_{c_{i}, l}=\left\{\begin{aligned} 1, & \text { if } c_{i} \text { can recive } r_{j} \text { or } i g w_{k} ; \\ & \text { where } l \in\left(r_{j} \cup i g w_{k}\right) . \\ 0, & \text { o.w. }\end{aligned}\right.$

$\Gamma_{r_{i}, i g w_{j}}= \begin{cases}1, & \text { if } r_{i} \text { can connect } i g w_{j} \\ 0, & \text { o.w. }\end{cases}$

The problem concerned in this paper is modelled as follows:

Maximize

$$
\begin{aligned}
\delta(G) & =\sum_{j \in\{1, \ldots o\}}\left[\sum_{i \in\{1, \ldots, n\}} \Gamma_{r_{i}, i g w_{j}}+\sum_{q \in\{1, \ldots, m\}} \sum_{l \in R U I G W} I_{c_{q}, l} \cdot \Gamma_{l, i g w_{j}}\right] \\
\phi(G) & =\sum_{i \in\{1, \ldots, m\}} \sum_{l \in R U I G W} I_{c_{i}, l}
\end{aligned}
$$

s.t.

$$
\begin{aligned}
& \sum_{j \in\{1, \ldots, o\}} \Gamma_{r_{i}, i g w_{j}} \leq 1, \forall i \in\{1, \ldots, n\} \\
& \sum_{l \in R \cup I G W} I_{c_{i}, l} \leq 1, \forall i \in\{1, \ldots, m\} \\
& \sum_{j \in\{1, \ldots, o\}} h_{r_{i}} \cdot \Gamma_{r_{i}, i g w_{j}} \leq D_{h o p}, \forall i \in\{1, \ldots, n\} \\
& \sum_{i \in\{1, \ldots, n\}} \lambda_{r_{i}, l} \leq R_{M R s}, \forall l \in R \cup I G W \\
& \sum_{i \in\{1, \ldots, m\}} \sum_{l \in R \cup I G W} I_{c_{i}, l} \cdot \Gamma_{l, i g w_{j}} \leq C_{M C s}, \forall j \in\{1, \ldots, w\} \\
& \sum_{i \in\{1, \ldots, n\}} \Gamma_{r_{i}, i g w_{j}} \leq C_{M R s}, \forall j \in\{1, \ldots, w\} \\
& \quad \Gamma_{l, i g w_{i}} \in\{0,1\}, \forall l \in R \cup I G W, i \in\{1, \ldots, w\} \\
& I_{c_{i}, r_{j}} \in\{0,1\}, \forall i \in\{1, \ldots, m\}, j \in\{1, \ldots, n\} ; h_{r_{i}} \in N, \forall i \in\{1, \ldots, n\} \\
& \quad \lambda_{r_{i}, l} \in\{0,1\}, \forall i \in\{1, \ldots, n\}, l \in R \cup I G W
\end{aligned}
$$

\section{A PSO APPROACH TO THE CONCERNED PROBLEM}

\section{A. Solutions Representation and Fitness Function}

The solution representation in the PSO approach is a placement of $n$ mesh routers in a two-dimensional $W \times H$ area, in which the lower-left corner is placed at the origin of an $x \times y$ plane, i.e., the $(x, y)$ positions of $n$ mesh routers are determined for candidate solutions. In PSO, each particle $k$ represents a candidate solution, which has three types of vectors as follows: 1) $X_{k}=\left(x_{k 1}, x_{k 2}, \ldots, x_{k(2 n)}\right)$ records the candidate solution of particle $k$, in which position $r_{i}$ is $\left(x_{k(2 i-1)}, x_{k(2 i)}\right)$, for $i \in\{1,2, \ldots$, $n\}$; 2) $P_{k}=\left(p_{k 1}, p_{k 2}, \ldots, p_{k(2 n)}\right)$ records the best solution of particle $k$ found so far; 3) $V_{k}=\left(v_{k 1}, v_{k 2}, \ldots, v_{k(2 n)}\right)$ records the velocity of particle $k$.

As all mesh routers are placed within the $W \times H$ deployment area, we require the following constraints:

$$
0 \leq x_{k(2 i-1)} \leq W, 0 \leq x_{k(2 i)} \leq H, \forall i \in\{1, \ldots, n\}
$$

To avoid drastic change of velocities, we require the following constraints:

$$
-V_{\text {max }} \leq v_{k(2 i-1)} \leq V_{\text {max }}, \quad-V_{\text {max }} \leq v_{k(2 i)} \leq V_{\max }, \forall i \in\{1, \ldots, n\}
$$


where $V_{\max }$ is a given constant value no more than $\max \{W, H\}$. $f\left(X_{k}\right)$ records the fitness of $X_{k} ; f\left(P_{k}\right)$ records the fitness of $P_{k}$. From the perspective of the whole swarm, the best position and fitness value found by all particles so far are recorded at each iteration: $P^{*}=\left(p_{1}^{*}, p_{2}^{*}, \ldots, p_{(2 n)}^{*}\right)$ and $f\left(P^{*}\right)$.

If particle $k$ has decided a placement $X_{k}$ of mesh routers, a topology graph $G_{k}$ corresponding to this placement can be created as explained in Section II. Note that the objective function of the concerned problem is multi-objective. In the past, a lot of methods existed to solve multi-objective problems [8]. This PSO in this paper applied a single fitness function by weighting the concerned two objectives as follows:

$$
\text { Maximize } \quad f\left(X_{k}\right)=\lambda \frac{\delta\left(G_{k}\right)}{m+n}+(1-\lambda) \frac{\phi\left(G_{k}\right)}{m}
$$

where

$$
\begin{aligned}
& \delta(G)=\sum_{j \in\{1, \ldots o\}}\left[\sum_{i \in\{1, \ldots, n\}} \Gamma_{r_{i}, i g w_{j}}+\sum_{q \in\{1, \ldots, m\}} \sum_{i \in R \cup l G W} I_{c_{q}, l} \times \Gamma_{l, i g w_{j}}\right] \\
& \phi(G)=\sum_{i \in\{1, \ldots, m\}} \sum_{l \in R \cup I G W} I_{c_{i}, l}
\end{aligned}
$$

Note that the $\lambda$ value is within $[0,1]$, which controls the balance between the two objectives. The denominator of each term of the fitness function is used for normalization.

\section{B. Position Updating}

The equation of updating velocity of particle $k$ at the $(t+1)$ th iteration is expressed as follows:

$$
V_{k}^{t+1}=\omega\left[V_{k}^{t}+c_{1} r_{1}\left(P_{k}^{t}-X_{k}^{t}\right)+c_{2} r_{2}\left(P^{*}-X_{k}^{t}\right)\right]
$$

where $c_{1}$ and $c_{2}$ are the cognitive learning rate and the social learning rate, and not relevant to mesh clients; $c=c_{1}+c_{2}>4$; $\omega=2 /\left|2-c-\sqrt{c^{2}-4 c}\right| ; r_{1}$ and $r_{2}$ are random number within $[0,1]$ and not relevant to mesh routers. The PSO with the above velocity updating equation is called a PSO with constriction coefficient [9]. Position of each particle $k$ at the $(t+1)$ th iteration is updated by the following formula:

$$
X_{k}^{t+1} \leftarrow X_{k}^{t}+V_{k}^{t+1}
$$

where $X_{k}^{t+1}$ is the updated value of position vector $X_{k}^{t}$ of particle $k . V_{k}^{t+1}$ is based on the best $P_{k}$ position of particle $k$ found so far, the global best position $P^{*}$, and the velocity $V_{k}^{t}$ of particle $k$ at the $t$ th iteration.

\section{Simulation Results}

This paper uses a similar scenario based on the previous research in [10]. The situation is as below: There are 4 Internet gateways, 16 mesh routers and 48 mesh clients in a $32 \times 32$ area. Each of Internet gateways and mesh routers covers a circle area with different radius which follows a uniform distribution $U(3,6)$. Each case has 10 instances, in which mesh clients are distributed in the deployment area according to a uniform distribution. Also, the four Internet gateways are distributed in four subareas of the deployment area based on [2].
We run 10 different instances 20 times with different initial position of clients. According to 20 run of fitness values, we draw statistic chart, box plot with the best, the third quartile, the median, the first quartile and the worst in Fig. 1.

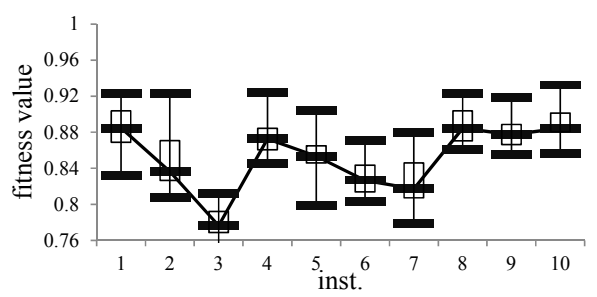

Fig. 1. Plots of fitness values and instance numbers

\section{CONCLUSIONS}

This paper presents a PSO approach for the router node placement problem in WMNs with Internet gateways subject to three QoS constraints. As the previous studies easily fell into local optima, the proposed PSO approach includes some delicate design to solve the local minima problem. Experiment results show that the proposed method not only gets better fitness, but also has a rapid convergence speed and global astringency.

\section{REFERENCE}

[1] A. Barolli, F. Xhafa, C. Sánchez, and M. Takizawa, "A study on the effect of mutation in genetic algorithms for mesh router placement problem in wireless mesh networks," in Proc. of 2011 International Conference on Complex, Intelligent and Software Intensive Systems (CISIS), pp. 32-39, 2011.

[2] F. Li, Y. Wang, X.-Y. Li, A. Nusairat, and Y. Wu, "Gateway placement for throughput optimization in wireless mesh networks," Mobile Networks and Applications, vol. 13, pp. 198-211, 2008.

[3] F. Xhafa, A. Barolli, C. Sánchez, and L. Barolli, "A simulated annealing algorithm for router nodes placement problem in Wireless Mesh Networks," Simulation Modelling Practice and Theory, vol. 19, pp. 2276-2284, 2011

[4] C.-C. Lin, "Dynamic router node placement in wireless mesh networks: A PSO approach with constriction coefficient and its convergence analysis," Information Sciences, vol. 232, pp. 294-308, 2013.

[5] B. Aoun, R. Boutaba, Y. Iraqi, and G. Kenward, "Gateway placement optimization in wireless mesh networks with QoS constraints," IEEE Journal on Selected Areas in Communications, vol. 24, pp. 2127-2136, 2006.

[6] M. Seyedzadegan, M. Othman, B. M. Ali, and S. Subramaniam, "Zerodegree algorithm for Internet gateway placement in backbone wireless mesh networks," Journal of Network and Computer Applications, vol. 36, pp. 1705-1723, 2013.

[7] J. Kennedy, "Particle swarm optimization," in Encyclopedia of Machine Learning, ed: Springer, pp. 760-766, 2010.

[8] R. L. Keeney and H. Raiffa, Decisions with Multiple Objectives: Preferences and Value Trade-offs, Cambridge University Press, 1993.

[9] M. Clerc and J. Kennedy, "The particle swarm-explosion, stability, and convergence in a multidimensional complex space," IEEE Transactions on Evolutionary Computation, vol. 6, pp. 58-73, 2002.

[10] F. Xhafa, C. Sánchez, and L. Barolli, "Genetic algorithms for efficient placement of router nodes in wireless mesh networks," in Proc. of 24th IEEE International Conference on Advanced Information Networking and Applications (AINA), pp. 465-472, 2010.

[11] H. A. Taha, Operations Research: An Introduction, Pearson/Prentice Hall, 2007. 